\title{
Washout Period
}

National Cancer Institute

\section{Source}

National Cancer Institute. Washout Period. NCI Thesaurus. Code C42872.

A period of time to allow one agent to clear the body before another agent can be safely introduced. Washout periods vary depending on the agents involved. 